\title{
Unusual Infrared Emission toward Sgr B2: Possible Planar $\mathbf{C}_{24}$
}

\author{
X. H. Chen ${ }^{1,2}$, F. Y. Xiang ${ }^{1}$, X. J. Yang ${ }^{1}$ and Aigen $\mathrm{Li}^{2}$ \\ 1 Department of Physics, Xiangtan University, 411105 Xiangtan, Hunan Province, China; \\ chenxh@smail.xtu.edu.cn, fyxiang@xtu.edu.cn, xjyang@xtu.edu.cn \\ 2 Department of Physics and Astronomy, University of Missouri, Columbia, MO 65211, USA; \\ lia@missouri.edu
}

\begin{abstract}
Interstellar graphene could be present in the interstellar medium (ISM), resulting from the photochemical processing of polycyclic aromatic hydrocarbon (PAH) molecules and/or collisional fragmentation of graphitic particles. Indeed, by comparing the observed ultraviolet (UV) extinction and infrared (IR) emission of the diffuse ISM with that predicted for graphene, as much as $\sim 2 \%$ of the total interstellar carbon could have been locked up in graphene without violating the observational constraints. While the possible detection of planar $\mathrm{C}_{24}$, a small piece of a graphene sheet, has been reported towards several Galactic and extragalactic planetary nebulae, graphene has not yet been detected in interstellar environments. In this work, we search for the characteristic IR features of $\mathrm{C}_{24}$ at $\sim 6.6,9.8,20 \mu \mathrm{m}$ toward Sgr B2, a high-mass star formation region, and find a candidate target toward R.A. $(\mathrm{J} 2000)=267^{\circ} .05855$ and Decl. $(\mathrm{J} 2000)=$ $-28^{\circ} .01479$ in Sgr B2 whose Spitzer/IRS spectra exhibit three bands peaking at $\sim 6.637$, 9.853 and $20.050 \mu \mathrm{m}$ which appear to be coincident with that of $\mathrm{C}_{24}$. Possible features of $\mathrm{C}_{60}$ are also seen in this region. The candidate region is a warm dust environment heated by massive stars or star clusters, associated with a WISE spot (a tracer of star-formation activity), close to the HII region candidate IRAS 17450-2759, and is surrounded by seven young stellar object candidates within $\sim 5^{\prime}$, suggesting that the creation and/or excitation of $\mathrm{C}_{24}$ could be related to star formation activities.
\end{abstract}

Key words: line: identification - ISM: lines and bands - ISM: molecules

\section{INTRODUCTION}

Carbon, with many allotropes known to be present in the interstellar medium (ISM; e.g., see Jäger et al. 2011), plays an important role in the physical and chemical evolution of the ISM (Henning \& Salama 1998). Several carbon nanostructures such as PAHs (e.g., Tielens. 2008), nanodiamonds (Guillois et al. 1999: Van Kerckhoven et al., 2002), fullerenes (e.g., Cami et al., 2010, Sellgren et al., 2010, GarcíaHernández et al., 2010, 2011b; Zhang \& Kwok, 2011, 2013; Berné et al., 2017) and their ions (e.g., Foing \& Ehrenfreund, 1994; Berné et al., 2013, Campbell et al., 2015, 2016; Strelnikov et al., 2015) are promising carries of many IR emission features seen in interstellar and circumstellar medium, although the exact identification still remains debated (see e.g., Kwok \& Zhang, 2011;, Yang et al., 2013. Rosenberg et al. 2014, Álvaro Galué \& Díaz Leines, 2017).

Graphene was first synthesized in the laboratory in 2004 by A.K. Geim and K.S. Novoselov (see Novoselov et al., 2004) for which they received the 2010 Nobel Prize in physics. García-Hernández 
et al. (2011a, 2012) first detected the unusual IR emission features at $\sim 6.6,9.8$, and $20 \mu \mathrm{m}$ in several Galactic and extragalactic planetary nebulae (PNe), which are coincident with the strongest transitions of a planar graphene sheet $\mathrm{C}_{24}$ theoretically predicted by Kuzmin \& Duley (2011) 1 More recently, Chen et al. (2017) have studied the UV absorption and IR emission of graphene $\mathrm{C}_{24}$. They estimated the abundance of graphene in the ISM to be $<5 \mathrm{ppm}$ of $\mathrm{C} / \mathrm{H}$ (i.e., $\sim 1.9 \%$ of the total interstellar C) by comparing the observed UV extinction and IR emission of the diffuse ISM with that predicted for graphene.

In principle, graphene could be present in the ISM as it could be formed from the photochemical processing of PAHs, which are abundant in the ISM, through a complete loss of their $\mathrm{H}$ atoms (e.g., see Berné \& Tielens, 2012). Chuvilin et al. (2010) showed experimentally that $\mathrm{C}_{60}$ could be formed from a graphene sheet. Berné \& Tielens (2012) further proposed that such a formation route could occur in space ${ }^{2}$ In these scenarios, the formation of $\mathrm{C}_{60}$ from graphene and the formation of graphene from PAHs are more likely to occur in regions rich in energetic UV photons. One such region is Sgr B2, a UV-rich high-mass star formation region. To this end, we search for the IR emission feature of $\mathrm{C}_{24}$ towards Sgr B2.

The star formation activity is overall deficient in the Central Molecular Zone (CMZ) of the Galaxy relative to its abundant gas (Guesten \& Downes, 1983; Morris \& Serabyn, 1996, Kauffmann et al. 2017), challenging the empirical rations between star formation rate and gas surface (Ginsburg et al., 2018). The distinctive physical and chemical parameters, such as pressure, temperature, velocity dispersion, abundances of elements, heating/cooling and chemical evolution, in the CMZ (Requena-Torres et al. 2006; Shetty et al. 2012, Grieco et al., 2015, Ginsburg et al., 2016; Henshaw et al., 2016; Tanaka et al. 2018, and references therein) are therefore of much interest. Most of the dust ridge clouds in the CMZ contain several thousand $M_{\odot}$ in stars or $<8 \%$ of star-formation efficiency (Barnes et al., 2017). Despite the active star formation in Sgr B2 (which contains actively forming star clusters, high-mass young stellar objects and many compact H II regions, e.g., Gaume et al., 1995: Higuchi et al., 2015), the overall cloud appears to be as inefficient as the other dust ridge clouds. We are thus eagerly to investigate the environment in the Sgr B2.

This paper is organized as follows. A brief description of the Spitzer Space Telescope spectroscopic data (Houck et al. 2004) we used is presented in Section 2, while the results of search are reported and discussed in Section 3. The main conclusion is given in Section 4.

\section{DATA DESCRIPTION}

The data analyzed here were obtained with the Infrared Spectrograph (IRS) on board the Spitzer Space Telescope and are publicly available $3^{3}$ (Houck et al. 2004, Werner et al. 2004). The data products for the short-low (SL) and long-low (LL) modules from the SSC pipeline (version 18.18) were used to produce the final merged spectra, making use of the four slits: SL2 $(5.21-7.56 \mu \mathrm{m})$ and SL1 $(7.57-14.28 \mu \mathrm{m})$ with resolution $R \sim 100$, and LL2 $(14.29-20.66 \mu \mathrm{m})$ and LL1 $(20.67-38.00 \mu \mathrm{m})$ with resolution $R \sim 100$ (see e.g., Simpson et al., 2007; Simpson, 2018). In particular, the SL2, SL1 and LL2 slits cover the wavelength regions of the most pronounced theoretical IR emission features of graphene $\mathrm{C}_{24}$ (i.e., at 6.6, 9.8 and $20 \mu \mathrm{m}$, see e.g., Martin et al., 1996; Kuzmin \& Duley, 2011; Chen et al., 2017, and references therein). We downloaded all the SL and LL data (103 pointing 4 ) toward Sgr B2 and used the software MATLAB to extract spectral information from the original data.

\footnotetext{
1 A small planar graphene sheet is essentially a fully dehydrogenated PAH molecule. The planar $\mathrm{C}_{24}$ graphene can be considered as coronene whose hydrogen atoms are completely lost, differing from the small fullerene $\mathrm{C}_{24}$. Bernstein et al. 2017) argued that the $11.3 \mu \mathrm{m}$ feature, commonly attributed to PAHs, could also arise from fullerene $\mathrm{C}_{24}$.

2 If there exists in the ISM a population of HAC-like nanoparticles with a mixed aromatic/aliphatic structure (e.g., see Kwok \& Zhang 2011), a complete loss of their H atoms could also convert HAC-like nanoparticles into graphene (e.g., see GarcíaHernández et al. 2010 2011a b . Also, graphene could be generated in the ISM from the exfoliation of graphite as a result of grain-grain collisional fragmentation. It is worth noting that graphite is thought to be a major dust component in the ISM (Draine) \& Lee 1984) and as mentioned earlier, presolar graphite grains have been identified in primitive meteorites.

3 Download from http://sha.ipac.caltech.edu/applications/Spitzer/SHA/

${ }^{4}$ We have searched for the IRS Enhanced data towards Sgr B2 within a radius of $\sim 1^{\circ}$ and obtained 103 pointings in total.
} 
Table 1 Information for Targets in Sgr B2

\begin{tabular}{ccccc}
\hline \hline Index & $\begin{array}{c}\text { R.A. (J2000) } \\
\text { (degree) }\end{array}$ & $\begin{array}{c}\text { Decl. (J2000) } \\
\text { (degree) }\end{array}$ & S/N & $\begin{array}{c}\sigma \\
(\mathrm{Jy})\end{array}$ \\
\hline 1 & 267.05851 & -28.01484 & 53 & 0.013 \\
2 & 267.05847 & -28.01476 & 53 & 0.013 \\
3 & 267.05857 & -28.01479 & 53 & 0.014 \\
4 & 267.05866 & -28.01478 & 53 & 0.013 \\
\hline
\end{tabular}

We searched for the simultaneous presence of all three emission features of graphene $\mathrm{C}_{24}$ at $\sim 6.6$, 9.8 and $20 \mu \mathrm{m}$ and found only four Sgr B2 pointings which exhibit all these features (see Table 1) 5 Table 1 lists the coordinates, the minimum signal-to-noise ratio $(\mathrm{S} / \mathrm{N})$ of the four slits reported from the original downloaded data, and noise level at $1 \sigma$. Because the maximum distance among the four points is $\sim 0.7^{\prime \prime}$, we took the mean spectrum toward the four pointings and the central coordinate of the new target is R.A. $(\mathrm{J} 2000)=267^{\circ} .05855$ and Decl. $(\mathrm{J} 2000)=-28^{\circ} .01479$. Figure 1 shows the three-color map to locate the target, where the blue, green, and red images are from the $21 \mu \mathrm{m}$ emission observed by the MidCourse Space Experiment (MSX; Price et al., 2001) and the 70 and $500 \mu \mathrm{m}$ emission from Herschel (Molinari et al., 2011), respectively.
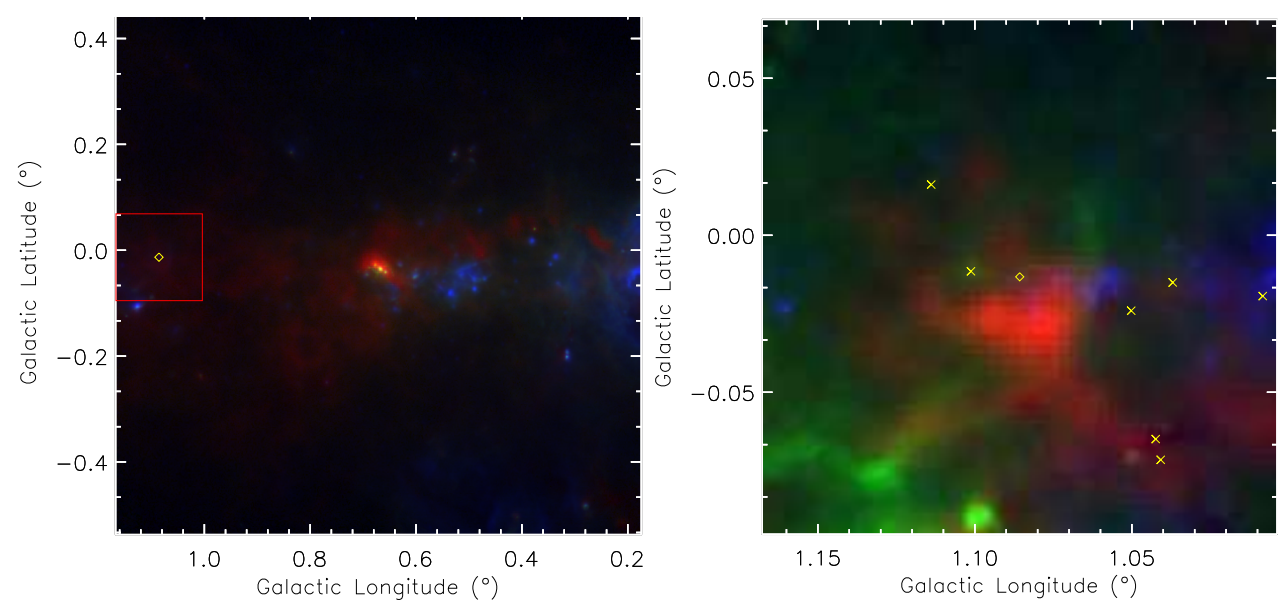

Fig. 1 Three-color images of the Sgr B2 region, where blue is the $21 \mu \mathrm{m}$ Band-E MSX image (Price et al., 2001), green is the $70 \mu \mathrm{m}$ image from Herschel InfraRed Galactic Plane Survey (Hi-GAL Molinari et al., 2011) which is taken with the Photodetector Array Camera and Spectrometer (Poglitsch et al., 2010) on Herschel Space Observatory (Pilbratt et al., 2010), and red is the $500 \mu \mathrm{m}$ image from Hi-GAL taken with the Spectral and Photometric Imaging Receiver (Griffin et al., 2010). The right panel is the close-up image of the red open box in the left panel.The diamond marks the target, and the crosses mark the possible YSOs (Robitaille et al. 2008; Yusef-Zadeh et al., 2009).

5 The PI for these Spitzer/IRS observations was Lee Armus with the Program ID of 1405, 1412, 1413, and 1419 in stare mode. All the four pointings are towards the object HDE216285 entitled by the PI. 


\section{RESULTS AND DISCUSSION}

\subsection{The Spectra}

We detected three IR emission features near the most pronounced theoretical IR emission features of planar $\mathrm{C}_{24}$ (i.e., at $\sim 6.6,9.8$ and $20 \mu \mathrm{m}$, see e.g., Martin et al., 1996; Kuzmin \& Duley, 2011; Chen et al. 2017, and references therein) towards all the four pointings (see Figure 2). In the following, we shall present the three components based on the mean spectrum in detail.

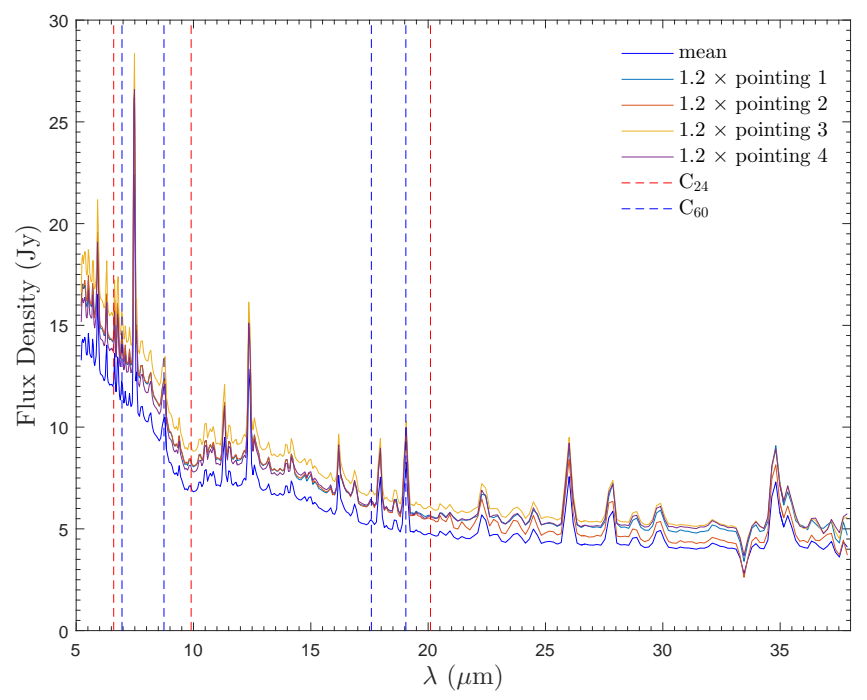

Fig. 2 The spectra of the four pointings with their flux density multiplied by 1.2 and the mean spectrum of the four pointings. The red and blue dashed lines indicate the possible $\mathrm{C}_{24}$ and $\mathrm{C}_{60}$ features, respectively.

We fit the spectra in terms of Gaussian profile (see Equation 1) in three windows centered at $\sim 6.64$, 9.85 and $20.05 \mu \mathrm{m}$ for $\mathrm{C}_{24}$, where a linear baseline is used in these windows with small wavelength span (i.e., $\lesssim 1 \mu \mathrm{m}$ ):

$$
F_{\lambda}=A_{0} \cdot \exp \left[-\left(\frac{\lambda-\lambda_{0}}{\Delta \lambda}\right)^{2}\right]+C_{1} \cdot \lambda+C_{2},
$$

where $A_{0}, C_{1}$ and $C_{2}$ are constants, $\lambda_{0}$ and $\Delta \lambda$ are respectively the peak wavelength and the width of the Gaussian profile. We find possible $\mathrm{C}_{24}$ emission toward Sgr B2 where the specified coordinates are described in Section 2 The mean spectrum of this candidate is shown in Figure 3 in detail. The results of the three components are catalogued in Table 2 Both $A_{0}$ of the three components are higher than 5 $\sigma$. The three central wavelengths are all close to those for the most pronounced theoretical IR emission features of planar $\mathrm{C}_{24}$ (i.e., at $\sim 6.6,9.8$ and $20 \mu \mathrm{m}$, see e.g., Martin et al., 1996, Kuzmin \& Duley, 2011; Chen et al., 2017, and references therein). That suggests that those three components are likely to originate from the planar, $\mathrm{C}_{24}$. The differences of the central wavelengths between the results reported in Table 2 and theoretical central wavelengths may be due to the well-known fact that DFT computation of $\mathrm{C}_{24}$ may not be precise in wavelength (Borowski, 2012).

The integrated intensity $(I)$ ratios for $\mathrm{C}_{24}$ calculated from the fitted line are $I_{1} / I_{2} \approx 2.2$ and $I_{1} / I_{3} \approx 4.0$, where subscripts denote component indexes. The observed $I_{1} / I_{2}$ here is comparable to the 

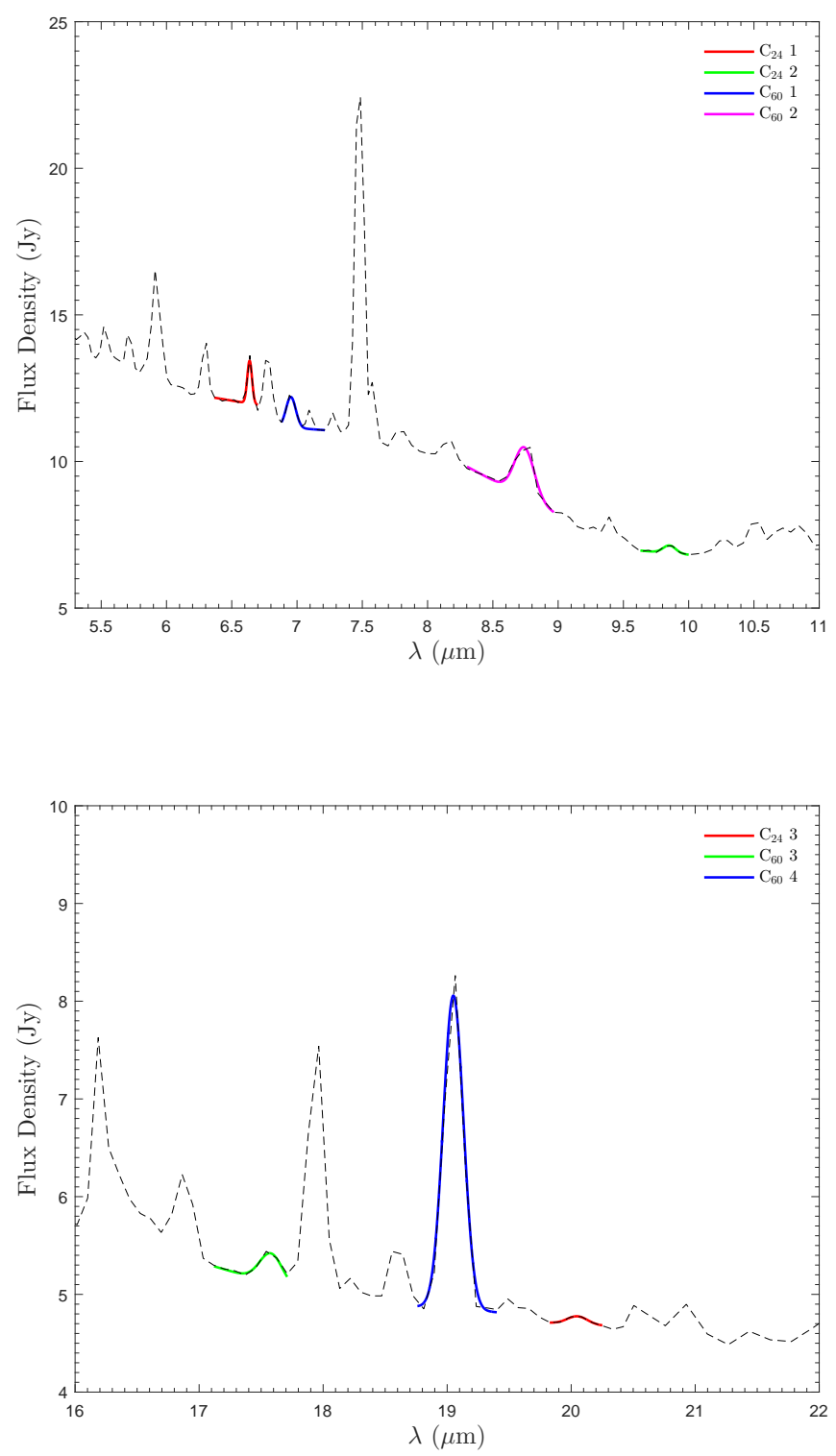

Fig. 3 The mean spectrum of the four pointings. The color spectra show the results of Gaussian fit for three components of $\mathrm{C}_{24}$ and four components of $\mathrm{C}_{60}$.

theoretical value of $\sim 1.8$, but the observed $I_{1} / I_{3}$ is higher than the theoretical value of $\sim 0.4$ predicted for $\mathrm{C}_{24}$ excited by the interstellar radiation field of the general diffuse ISM (Chen et al., 2017). The band ratios are sensitive to the starlight spectral shape (i.e., "hardness") and, to a less degree, to the starlight intensity (see Draine \& Li, 2001). An exact match would require detailed IR emission modeling of $\mathrm{C}_{24}$ in Sgr B2 in the future. 
Table 2 Gaussian Fits to Three Possible Components of $\mathrm{C}_{24}$

\begin{tabular}{cccccc}
\hline \hline Components & $\begin{array}{c}\lambda_{0} \\
(\mu \mathrm{m})\end{array}$ & $\begin{array}{c}\Delta \lambda \\
(\mu \mathrm{m})\end{array}$ & $\begin{array}{c}A_{0} \\
(\mathrm{Jy})\end{array}$ & $\begin{array}{c}C_{1} \\
\left(\mathrm{Jy}_{\mu \mathrm{m}}-1\right)\end{array}$ & $\begin{array}{c}C_{2} \\
(\mathrm{Jy})\end{array}$ \\
\hline 1 & $6.637 \pm 0.001$ & $0.026 \pm 0.001$ & $1.463 \pm 0.025$ & $-0.747 \pm 0.083$ & $16.930 \pm 0.540$ \\
2 & $9.853 \pm 0.001$ & $0.067 \pm 0.002$ & $0.260 \pm 0.005$ & $-0.383 \pm 0.022$ & $10.650 \pm 0.210$ \\
3 & $20.050 \pm 0.000$ & $0.116 \pm 0.002$ & $0.082 \pm 0.001$ & $-0.711 \pm 0.003$ & $6.119 \pm 0.066$ \\
\hline
\end{tabular}

Table 3 Gaussian Fits to Four Possible Components of $\mathrm{C}_{60}$

\begin{tabular}{|c|c|c|c|c|c|}
\hline Components & $\begin{array}{c}\lambda_{0} \\
(\mu \mathrm{m})\end{array}$ & $\begin{array}{c}\Delta \lambda \\
(\mu \mathrm{m})\end{array}$ & $\begin{array}{l}A_{0} \\
(\mathrm{Jy})\end{array}$ & 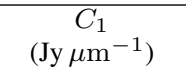 & $\begin{array}{l}C_{2} \\
\text { (Jy) }\end{array}$ \\
\hline 1 & $6.953 \pm 0.001$ & $0.053 \pm 0.001$ & $1.047 \pm 0.022$ & $-0.321 \pm 0.102$ & $13.380 \pm 0.730$ \\
\hline 2 & $8.742 \pm 0.001$ & $0.107 \pm 0.002$ & $1.696 \pm 0.024$ & $-2.364 \pm 0.056$ & $29.450 \pm 0.480$ \\
\hline 3 & $17.580 \pm 0.000$ & $0.130 \pm 0.003$ & $0.304 \pm 0.008$ & $-0.373 \pm 0.023$ & $11.680 \pm 0.390$ \\
\hline 4 & $19.050 \pm 0.000$ & $0.115 \pm 0.001$ & $3.029 \pm 0.011$ & $-0.090 \pm 0.019$ & $6.564 \pm 0.354$ \\
\hline
\end{tabular}

Because the $\mathrm{C}_{24}$ features have been detected in conjunction with fullerene features in Galactic and extra-galactic PNes (García-Hernández et al. 2011a. 2012), we also fit the spectrum by Gaussian profiles (see Equation 1) in four windows centered at $\sim 7.0,8.8,17.4$ and $18.9 \mu \mathrm{m}$ for $\mathrm{C}_{60}$ (see Figure 3). The results are cataloged in Table 3 . The $\mathrm{C}_{60}$ integrated intensity ratios are $I_{1} / I_{4} \sim 0.16, I_{2} / I_{4} \sim 0.52$, and $I_{3} / I_{4} \sim 0.11$. These band ratios differ from those of PNe (Sellgren et al., 2010) and reflection nebulae (Bernard-Salas et al. 2012). But this could be simply due to the fact that the excitation condition of $\mathrm{C}_{60}$ varies from our target to those of PNe and reflection nebulae (Iglesias-Groth et al., 2011). Both the intensity and "hardness" of the starlight radiation fields to which $\mathrm{C}_{60}$ is exposed would affect the temperature distribution of $\mathrm{C}_{60}$. Note that $\mathrm{C}_{60}$, like the $\mathrm{C}_{24}$ graphene, undergoes single-photon heating and the maximum temperature it attains is sensitive to the spectral shape of the illuminating starlight (see Draine \& Li, 2001).

The coexistence of $\mathrm{C}_{24}$ and $\mathrm{C}_{60}$ further confirms that the IR emission at $\sim 6.637,9.853$ and $20.050 \mu \mathrm{m}$ may originate from planar $\mathrm{C}_{24}$. García-Hernández et al. (2011a) propose that shocks which are driven by strong stellar winds, can trigger HACs' processing. Fullerenes, possibly planar $\mathrm{C}_{24}$ molecules, and other complex aromatic and aliphatic species, could evolve from the vaporization of HACs.

As discussed above, we refer to the candidate carrier of the three components (centered at at $\sim 6.637,9.853$ and $20.050 \mu \mathrm{m}$ ) as planar $C_{24}$. Further observations, especially with higher spectral resolution, and further experimental research and theoretical computation are required to confirm this.

\subsection{Relation with Star-Formation Activity}

The excitation of $\mathrm{C}_{24}$ requires UV photons. As illustrated in Figure 2 of Chen et al. (2017), $\mathrm{C}_{24}$ mostly absorbs in the far-UV. Therefore, in regions with intense star-formation activities, the excitation of $\mathrm{C}_{24}$ is naturally expected to occur. Also, the creation of $\mathrm{C}_{24}$ from PAHs, $\mathrm{HAC}$ and/or graphite is also more likely to occur in UV-rich regions.

In addition to the $21 \mu \mathrm{m}, 70$ and $500 \mu \mathrm{m}$ images (see Figure 1, Price et al., 2001; Molinari et al. 2011), we also provide high-sensitivity mid-infrared images from the Wide-field Infrared Survey Explorer (WISE, see Figure 4, Wright et al., 2010) to indicate the protostar activity. The candidate YSOs are superposed in these two figures. The 21 and $70 \mu \mathrm{m}$ emission show the warm dust heated by nearby massive stars or star clusters; and the far-infrared emission (e.g. at $500 \mu \mathrm{m})$ that is from the cold dust, shows the locations of the dense molecular clouds that have few indicators of active star formation (Simpson, 2018, and references therein). As stated in Wright et al. (2010), the excesses at $12 \mu \mathrm{m}$ and $22 \mu \mathrm{m}$ bands are indicator of star formation activity. Both Figures 1 and 4 indicate that the target is probably impacted by star formation. The former one delineates a star-forming region where the warm 
dust is heated by nearby massive stars or star clusters and is surrounded by cold dust. The excesses at $12 \mu \mathrm{m}$ and $22 \mu \mathrm{m}$ bands in the latter one also imply that the source is probably impacted by star formation.

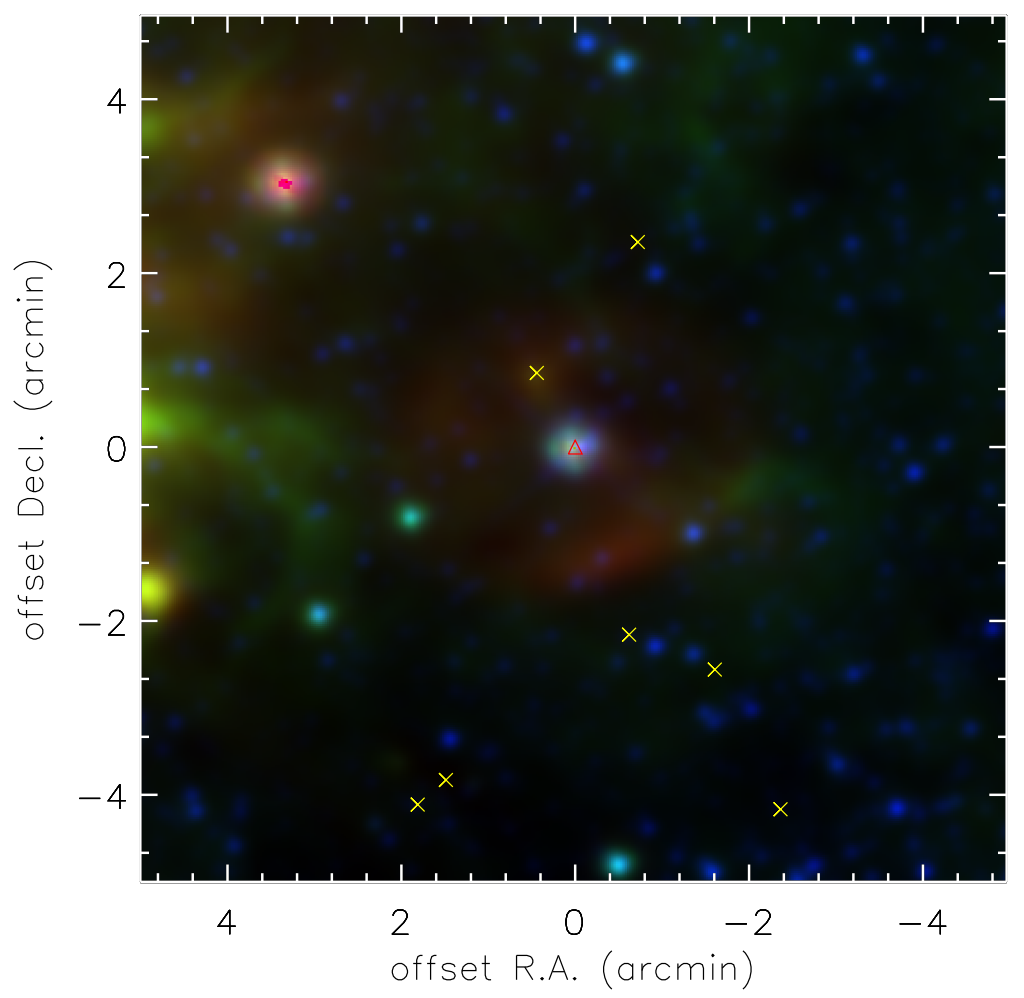

Fig. 4 Background false-color map centered on the target (red triangle) at R.A. $(\mathrm{J} 2000)=$ $267^{\circ} .05855$ and Decl. $\left.(\mathrm{J} 2000)=-28^{\circ} .01479\right)$ with seven YSO candidates superimposed (yellow crosses, Robitaille et al. 2008; Yusef-Zadeh et al., 2009), where blue is the WISE $4.6 \mu \mathrm{m}$, green is $12 \mu \mathrm{m}$, and red is $22 \mu \mathrm{m}$ data.

The target region where $\mathrm{C}_{24}$ is seen is more likely associated with HII regions than PNe. The IRAS source (with an angular resolution of $\sim 0.75^{\prime}-3.0^{\prime}$ ), IRAS 17450-2759, of 13.7" away from the target shows $F_{12} / F_{25}=0.64, F_{25} / F_{60}=0.06, F_{60} / F_{12}=27.58, F_{100} / F_{12}=112.74, F_{100} / F_{25}=71.94$ and $F_{100} / F_{60}=4.09$, where $F_{12}, F_{25}, F_{60}$, and $F_{100}$ denote the flux densities at $12,25,60$ and $100 \mu \mathrm{m}$. The corresponding flux qualities are $Q_{12}=3, Q_{25}=2, Q_{60}=1$, and $Q_{100}=3.6$ respectively. Both of them are conformity with criteria of HII regions which are excited by embedded high mass stars (except $Q_{60}>1$ required in some criteria, see Yan et al., 2018) rather than PNe's property (Kohoutek, 2001).

We have also searched for YSOs from SimBad ${ }^{7}$ within $5^{\prime}$ to the target in this work. Seven YSO candidates ${ }^{8}$ (Robitaille et al., 2008, Yusef-Zadeh et al., 2009) are surrounding the target, indicating that the candidate planar $\mathrm{C}_{24}$ emission is likely to associate with star formation activity.

6 The flux quality values of 1,2 , and 3 represent an upper limit, moderate quality, and high quality, respectively (see https: //heasarc.gsfc.nasa.gov/W3Browse/all/iraspsc.html).

Thtp://simbad.u-strasbg.fr/simbad/sim-fcoo

8 Include SSTGC 891214, SSTGC 899543, SSTGC 909173, 2MASS J17480460-2805032, 2MASS J17480762-2803267 2MASS J17481157-2803027 from Yusef-Zadeh et al. (2009), and SSTGLMC G001.0423-00.0650 from Robitaille et al. (2008). 
In addition, the 4.6, 12 and $22 \mu \mathrm{m}$ filters from WISE data include the continuum emission from ultrasmall grains which undergo stochastic heating (Wright et al. 2010). These nano-sized ultrasmall grains, if they are carbonaneous in nature like HAC, their collisional fragmentation and full dehydrogenation, triggered by UV photons and/or shockwaves from star formation activities, could result in the creation of $\mathrm{C}_{24}$. Therefore, it is not surprising that $\mathrm{C}_{24}$ is related to star formation activity.

Finally, we note that it would potentially be useful to compare the physical and chemical conditions (e.g., the UV starlight intensities, the hydrogen and electron number densities, and the gas temperature) of the four pointings in which $\mathrm{C}_{24}$ is possibly present with that of the other pointings in which $\mathrm{C}_{24}$ is not seen. However, it is difficult to obtain a complete census of the YSOs and their initial mass functions in these regions. Also, the UV extinction prevents us from an accurate direct measure of the UV starlight intensities even if the UV photometric or imaging data are available (e.g., from HST). Therefore, we prefer not to make comparisons between the environments of the four pointings and the other pointings.

\section{SUMMARY}

PAHs, one of graphene precursor, are widespread in star-forming environments. Inspired by the possible detection of the $\mathrm{C}_{24}$ emission features at $\sim 6.6,9.8,20 \mu \mathrm{m}$ in several Galactic and extragalactic PNe, and theoretical endeavor recently about $\mathrm{C}_{24}$ in ISM, we have searched for the characteristic IR emission features of $\mathrm{C}_{24}$ toward the high-mass star formation region Sgr B2. We detected IR emission, with three peak wavelengths of $\sim 6.637,9.853$ and $20.050 \mu \mathrm{m}$ toward R.A. $(\mathrm{J} 2000)=267^{\circ} .05855$ and Decl. $(\mathrm{J} 2000)=-28^{\circ} .01479$ in Sgr B2. These three wavelengths are all very close to the most pronounced three characteristic IR emission features of $\mathrm{C}_{24}$. These detected features are also companied with the characteristic IR emission bands of possibly $\mathrm{C}_{60}$.

The three-color $(21,70$ and $500 \mu \mathrm{m})$ images indicate that the target is probably in the warm dust environment which is heated by nearby massive stars or star clusters. The WISE false-color map suggests that the target source is associated with a WISE spot with excesses at $12 \mu \mathrm{m}$ and $22 \mu \mathrm{m}$, a tracer of star-formation activity. The nearest IRAS source (IRAS 17450-2759) is 13.7" away, and this IRAS source is a HII region candidate. The target source is also surrounded by seven YSO candidates within $5^{\prime}$. All these suggest that the IR emission of $\mathrm{C}_{24}$ could be powered by star formation activity.

\section{ACKNOWLEDGMENTS}

We thank the anonymous referee for his/her very helpful suggestions and comments which substantially improved the quality of this work. This research is supported by Hunan Provincial Innovation Foundation for Postgraduates CX2015B213, and by the Joint Research Funds in Astronomy (U1531108, U1731106 and U1731110) under cooperative agreement between the National Natural Science Foundation of China and Chinese Academy of Sciences, and in part by NSFC U1731107.

\section{References}

Alata, I., Cruz-Diaz, G. A., Muñoz Caro, G. M., \& Dartois, E. 2014, A\&A, 569, A119

Allamandola, L. J., Tielens, A. G. G. M., \& Barker, J. R. 1989, ApJS, 71, 733

Álvaro Galué, H., \& Díaz Leines, G. 2017, Physical Review Letters, 119, 171102

Bernard-Salas, J., Cami, J., Peeters, E., et al. 2012, ApJ, 757, 41

Barnes, A. T., Longmore, S. N., Battersby, C., et al. 2017, MNRAS, 469, 2263

Berné, O., Cox, N. L. J., Mulas, G., \& Joblin, C. 2017, A\&A, 605, L1

Berné, O., Mulas, G., \& Joblin, C. 2013, A\&A, 550, L4

Berné, O., \& Tielens, A. G. G. M. 2012, Proceedings of the National Academy of Science, 109, 401

Bernstein, L. S., Shroll, R. M., Lynch, D. K., \& Clark, F. O. 2017, ApJ, 836, 229

Borowski, P. 2012, Journal of Physical Chemistry A, 116, 3866

Cami, J., Bernard-Salas, J., Peeters, E., \& Malek, S. E. 2010, Science, 329, 1180

Campbell, E. K., Holz, M., Gerlich, D., \& Maier, J. P. 2015, Nature, 523, 322 
Campbell, E. K., Holz, M., Maier, J. P., et al. 2016, ApJ, 822, 17

Chen, X. H., Li, A., \& Zhang, K. 2017, ApJ, 850, 104

Chuvilin, A., Kaiser, U., Bichoutskaia, E., Besley, N. A., \& Khlobystov, A. N. 2010, Nature Chemistry, 2,450

Draine, B. T., \& Lee, H. M. 1984, ApJ, 285, 89

Draine, B. T., \& Li, A. 2001, ApJ, 551, 807

Foing, B. H., \& Ehrenfreund, P. 1994, Nature, 369, 296

García-Hernández, D. A., Iglesias-Groth, S., Acosta-Pulido, J. A., et al. 2011, ApJ, 737, L30

García-Hernández, D. A., Rao, N. K., \& Lambert, D. L. 2011, ApJ, 739, 37

García-Hernández, D. A., Manchado, A., García-Lario, P., et al. 2010, ApJ, 724, L39

García-Hernández, D. A., Villaver, E., García-Lario, P., et al. 2012, ApJ, 760, 107

Gaume, R. A., Claussen, M. J., de Pree, C. G., Goss, W. M., \& Mehringer, D. M. 1995, ApJ, 449, 663

Ginsburg, A., Bally, J., Barnes, A., et al. 2018, ApJ, 853, 171

Ginsburg, A., Henkel, C., Ao, Y., et al. 2016, A\&A, 586, A50

Grieco, V., Matteucci, F., Ryde, N., Schultheis, M., \& Uttenthaler, S. 2015, MNRAS, 450, 2094

Griffin, M. J., Abergel, A., Abreu, A., et al. 2010, A\&A, 518, L3

Guesten, R., \& Downes, D. 1983, A\&A, 117, 343

Guillois, O., Ledoux, G., \& Reynaud, C. 1999, ApJ, 521, L133

Henning, T., \& Salama, F. 1998, Science, 282, 2204

Henshaw, J. D., Longmore, S. N., Kruijssen, J. M. D., et al. 2016, MNRAS, 457, 2675

Higuchi, A. E., Hasegawa, T., Saigo, K., Sanhueza, P., \& Chibueze, J. O. 2015, ApJ, 815, 106

Houck, J. R., Roellig, T. L., van Cleve, J., et al. 2004, ApJS, 154, 18

Iglesias-Groth, S., Cataldo, F., \& Manchado, A. 2011, MNRAS, 413, 213

Jäger, C., Posch, T., Mutschke, H., et al. 2011, The Molecular Universe, 280, 416

Kauffmann, J., Pillai, T., Zhang, Q., et al. 2017, A\&A, 603, A89

Kim, J. H., Im, M., Lee, H. M., et al. 2012, ApJ, 760, 120

Kohoutek, L. 2001, A\&A, 378, 843

Kwok, S., Volk, K., \& Bernath, P. 2001, ApJ, 554, L87

Kwok, S., \& Zhang, Y. 2011, Nature, 479, 80

Kuzmin, S., \& Duley, W. W. 2011, arXiv:1103.2989

Martin, J. M. L., El-Yazal, J., \& François, J.-P. 1996, Chemical Physics Letters, 255, 7

Molinari, S., Bally, J., Noriega-Crespo, A., et al. 2011, ApJ, 735, L33

Morris, M., \& Serabyn, E. 1996, ARA\&A, 34, 645

Novoselov, K. S., Geim, A. K., Morozov, S. V., et al. 2004, Science, 306, 666

Pendleton, Y. J., \& Allamandola, L. J. 2002, ApJS, 138, 75

Pilbratt, G. L., Riedinger, J. R., Passvogel, T., et al. 2010, A\&A, 518, L1

Poglitsch, A., Waelkens, C., Geis, N., et al. 2010, A\&A, 518, L2

Price, S. D., Egan, M. P., Carey, S. J., Mizuno, D. R., \& Kuchar, T. A. 2001, AJ, 121, 2819

Requena-Torres, M. A., Martín-Pintado, J., Rodríguez-Franco, A., et al. 2006, A\&A, 455, 971

Robitaille, T. P., Meade, M. R., Babler, B. L., et al. 2008, AJ, 136, 2413

Rosenberg, M. J. F., Berné, O., \& Boersma, C. 2014, A\&A, 566, L4

Sellgren, K., Werner, M. W., Ingalls, J. G., et al. 2010, ApJ, 722, L54

Shetty, R., Beaumont, C. N., Burton, M. G., Kelly, B. C., \& Klessen, R. S. 2012, MNRAS, 425, 720

Simpson, J. P. 2018, ApJ, 857, 59

Simpson, J. P., Colgan, S. W. J., Cotera, A. S., et al. 2007, ApJ, 670, 1115

Strelnikov, D., Kern, B., \& Kappes, M. M. 2015, A\&A, 584, A55

Tanaka, K., Nagai, M., Kamegai, K., Iino, T., \& Sakai, T. 2018, ApJS, 236, 40

Tielens, A. G. G. M. 2008, ARA\&A, 46, 289

Van Kerckhoven, C., Tielens, A. G. G. M., \& Waelkens, C. 2002, A\&A, 384, 568

Werner, M. W., Roellig, T. L., Low, F. J., et al. 2004, ApJS, 154, 1

Wright, E. L., Eisenhardt, P. R. M., Mainzer, A. K., et al. 2010, AJ, 140, 1868

Yan, Q.-Z., Xu, Y., Walsh, A. J., et al. 2018, MNRAS, 476, 3981 
Yang, X. J., Glaser, R., Li, A., \& Zhong, J. X. 2013, ApJ, 776, 11

Yusef-Zadeh, F., Hewitt, J. W., Arendt, R. G., et al. 2009, ApJ, 702, 178

Zhang, Y., \& Kwok, S. 2011, ApJ, 730, 126

Zhang, Y., \& Kwok, S. 2013, Earth, Planets, and Space, 65, 1069 